\title{
Adenovirus adenine nucleotide translocator-2 shRNA effectively induces apoptosis and enhances chemosensitivity by the down-regulation of ABCG2 in breast cancer stem-like cells
}

\author{
Ji-Young Jang ${ }^{1}$, Min-Kyoung Kim ${ }^{1}$, \\ Yoon-Kyung Jeon ${ }^{1}$, Yoon-Ki Joung ${ }^{2}$, \\ Ki-Dong Park ${ }^{3}$ and Chul-Woo Kim ${ }^{1,4}$ \\ ${ }^{1}$ Tumor Immunity Medical Research Center \\ Cancer Research Institute \\ Seoul National University College of Medicine \\ Seoul 110-799, Korea \\ ${ }^{2}$ Center for Biomaterials \\ Korea Institute of Science and Technology \\ Seoul 136-791, Korea \\ ${ }^{3}$ Department of Molecular Science and Technology \\ Ajou University \\ Suwon 443-749, Korea \\ ${ }^{4}$ Corresponding author: Tel, 82-2-3668-7444; \\ Fax, 82-2-3676-7449; E-mail, cwkim @ snu.ac.kr \\ http://dx.doi.org/10.3858/emm.2012.44.4.019
}

Accepted 16 December 2011

Available Online 26 December 2011

Abbreviations: ANT2, adenine nucleotide translocator-2; CSCs, cancer stem cells; RNAi, RNA interference; shRNA, short-hairpin RNA; SP, sub-population

\begin{abstract}
Cancer stem cells (CSCs) are resistant to chemo- and radio-therapy, and can survive to regenerate new tumors. This is an important reason why various anti-cancer therapies often fail to completely control tumors, although they kill and eliminate the bulk of cancer cells. In this study, we determined whether or not adenine nucleotide translocator-2 (ANT2) suppression could also be effective in inducing cell death of breast cancer stem-like cells. A sub-population (SP; $\mathrm{CD}_{4}^{+}{ }^{+}$) CD24) of breast cancer cells has been reported to have stem/progenitor cell properties. We utilized the adeno-ANT2 shRNA virus to inhibit ANT2 expression and then observed the treatment effect in a SP of breast cancer cell line. In this study, MCF7, MDA-MB-231 cells, and breast epithelial cells (MCF10A) mesenchymally-
\end{abstract}

transdifferentiated through E-cadherin knockdown were used. ANT2 expression was high in both stem-like cells and non-stem-like cells of MCF7 and MDA-MB-231 cells, and was induced and up-regulated by mesenchymal transdifferentiation in MCF10A cells (MCF10A ${ }^{\mathrm{EMT}}$ ). Knockdown of ANT2 by adeno-shRNA virus efficiently induced apoptotic cell death in the stem-like cells of MCF7 and MDA-MB-231 cells, and MCF10A ${ }^{\mathrm{EMT}}$. Stemlike cells of MCF7 and MDA-MB-231, and MCF10A ${ }^{\text {EMT }}$ cells exhibited increased drug (doxorubicin) resistance, and expressed a multi-drug resistant related molecule, ABCG2, at a high level. Adeno-ANT2 shRNA virus markedly sensitized the stem-like cells of MCF7 and MDA-MB-231, and the MCF10A ${ }^{\mathrm{EMT}}$ cells to doxorubicin, which was accompanied by down-regulation of ABCG2. Our results suggest that ANT2 suppression by adeno-shRNA virus is an effective strategy to induce cell death and increase the chemosensitivity of stem-like cells in breast cancer.

Keywords: ABCG2 protein, human; adenine nucleotide translocator 2; drug therapy, combination; gene therapy; neoplastic stem cells; RNA, small interfering

\section{Introduction}

Although conventional chemotherapy kills most tumor cells, it is believed to leave some cells behind, which have proposed to serve as the source of recurrence. The cells that tend to remain are thought to be cancer stem cells (CSCs) (Dean et al., 2005). These CSCs have been found to be resistant to many current cancer treatments, including chemoand radio-therapy (Wulf et al., 2001; Liu et al., 2006; Phillips et al., 2006; Hermann et al., 2007; Todaro et al., 2007; Ma et al., 2008). Further, they demonstrate a capacity for self-renewal and generation of heterogeneous progeny (Al-Hajj and Clarke, 2004; Ponti et al., 2005; Vermeulen et al., 2008). These cells can be distinguished from their non-tumorigenic counterparts by a specific cell surface marker profile. Breast cancer cells with high levels of CD44 (CD44 $\left.{ }^{\text {high }}\right)$ and absent or low CD24 (CD24 $4^{\text {neglow }}$ ) expression have 
functional characteristics of CSCs (Thompson et al., 1992; Gordon et al., 2003; Charafe-Jauffret et al., 2006; Elstrodt et al., 2006). Additionally, CD24 is expressed in differentiated cells, whereas CD44 is expressed in more progenitor-like cells. The CD44 level has been found to be directly correlated with metastasis in breast cancer. Specifically, it has been reported that breast cells of the CD44+/CD24phenotype express genes that are involved in cell motility and angiogenesis, are more mesenchymal, motile, and are predominately estrogen receptornegative (Sheridan et al., 2006; Shipitsin et al., 2007). Hoechst33342 dye efflux, suspension sphere assays, and serial transplantation have also been proposed methods for identifying and separating CSCs from solid tumors (Pearce and Bonnet, 2007). However, the most effective method of identifying these cells, which may be morphologically indistinct from the bulk of the tumor, has been through differential cell surface protein expression.

CSCs are more resistant to chemo- and radiotherapy than non-CSCs (Wulf et al., 2001; Dean et al., 2005; Liu et al., 2006; Phillips et al., 2006; Hermann et al., 2007; Todaro et al., 2007; Ma et al., 2008), they exhibit multi-drug resistance (MDR) properties, and ABCG2/BCRP is particularly overexpressed in CSCs (Dean et al., 2005). The ATP-binding cassette membrane transporter, $A B C G 2 / B C R P$, functions as an energy-dependent efflux pump, and the expression of ABCG2 has been associated with multi-drug resistance. More significantly, ABCG2 has been identified as the molecular determinant for the sub-population (SP; CD44+/CD24-) phenotype and has been postulated as a universal stem cell marker (Zhou et al., 2001; Schinkel and Jonker, 2003). Moreover, the induction of an epithelial-mesenchymal transition (EMT) in normal or neoplastic mammary epithelial cell populations has been shown to result in the enrichment of cells with stem-like properties (Morel et al., 2008). We demonstrated herein that immortalized breast epithelial cells experimentallyinduced into an EMT also exhibited an increased ANT2 expression.

ANT2 is specifically expressed in undifferentiated cells or tissues that are able to proliferate and regenerate (e.g., lymphocytes, kidney, and liver; Gross et al., 1999; Suliman et al., 2001; Jang et al., 2008). The expression of ANT2 was recently shown to be up-regulated in several hormone-dependent cancers (Doerner et al., 1997), and the induction of ANT2 expression in cancer cells has been directly associated with glycolytic metabolisms, raising a question regarding the role of ANT2 during carcinogenesis (Stepien et al., 1992; Faure Vigny et al., 1996; Luciakova et al., 2003; Chevrollier et al., 2005a, 2005b, 2005c; Le Bras et al., 2006). Mesenchymal stem cells (MSCs) also require a high energy supply, and in particular, glycolytic metabolism and oxidative phosphorylation are increased, requiring increased expression of related genes such as ANT2 (Kong et al., 2011).

According to our previous study, ANT2 suppression by a DNA vector based RNA interference approach expressing short hairpin RNA (shRNA) resulted in ATP depletion from breast cancer cells and induced cell death (Jang et al., 2008). Based on this background, we hypothesized that the knockdown of ANT2 using ANT2 shRNA systems might be able to induce apoptotic cell death and increase chemosensitivity in breast cancer stem-like cells. Herein, we show that adno-ANT2 shRNA virus is also cytotoxic to breast cancer stem-like cells, and that sensitizing the breast cancer stem-like cells to chemotherapeutic agents would be effective in the control of breast cancer.

A
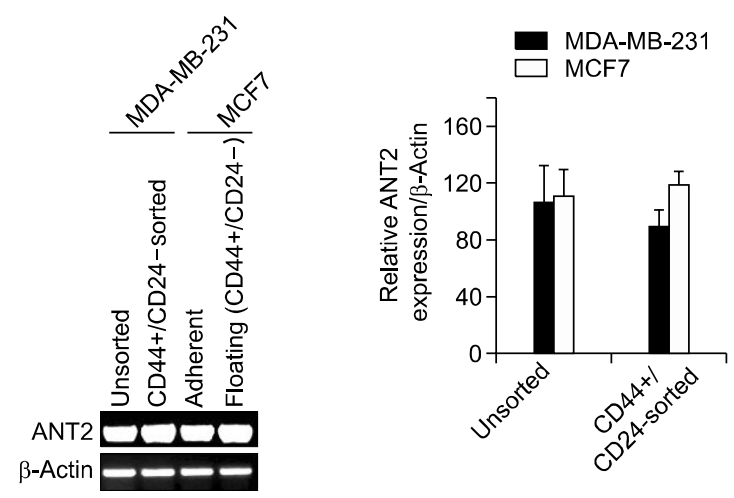

B
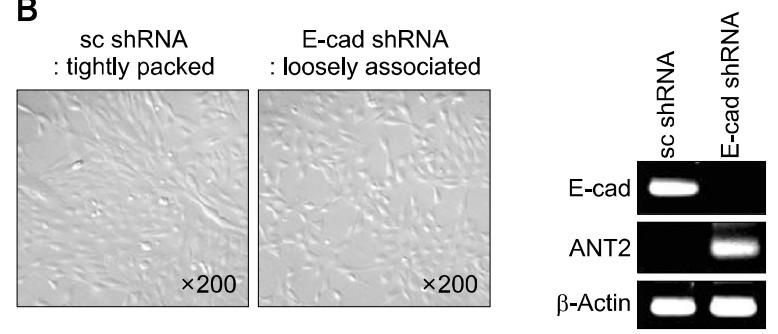

Figure 1. ANT2 is highly expressed in both cancer stem-like cells and non-cancer stem-like cells of breast cancer. (A) CD44+/CD24- fractions were sorted from MDA-MB-231 and MCF7 cells using the MACS. The sorting purities were confirmed by FACS analysis. RT-PCR to detect the level of ANT2 expression in unsorted and CD44+/CD24- sorted cells. Total RNA was extracted from respective cells and subjected to RT-PCR using specific primers for human ANT2 or $\beta$-actin (internal control). These data were confirmed by the GRT-PCR and normalized by $\beta$-actin mRNA. (B) MCF10A cells were transfected with scramble shRNA or E-cadherin shRNA (each $1 \mu \mathrm{g}$ ). EMT-associated morphology was detected by microscope, and the change in ANT2 expression was detected by RT-PCR. 
A

CD44+/CD24-sorted MDA-MB-231

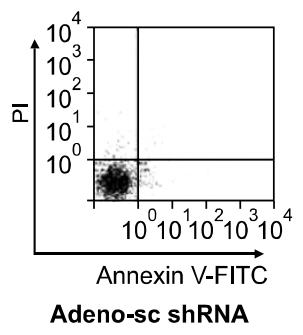

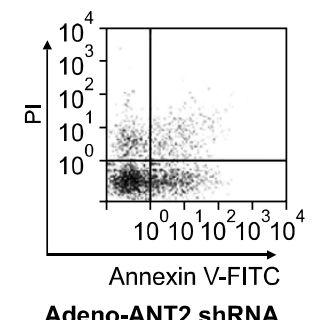

Adeno-ANT2 shRNA

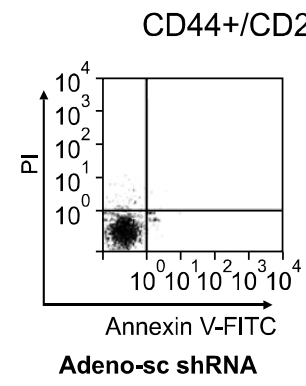

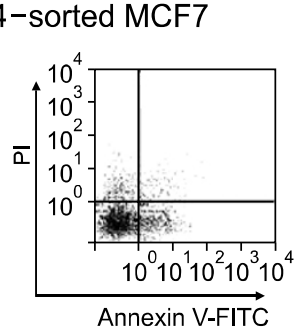

Adeno-ANT2 shRNA

B

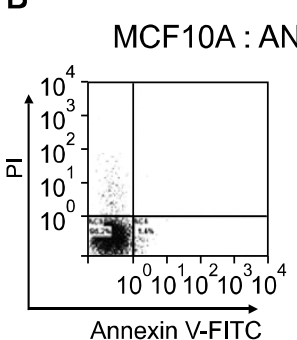

Adeno-sc shRNA

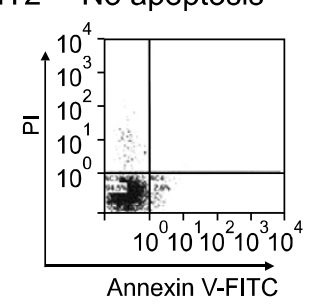

Adeno-ANT2 shRNA

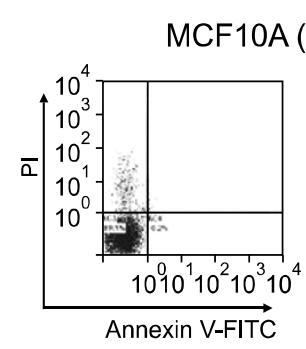

Adeno-sc shRNA

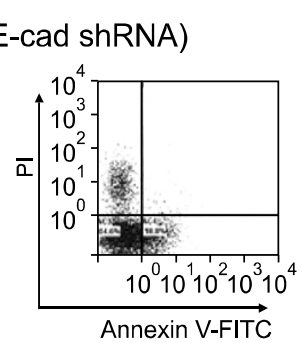

Adeno-ANT2 shRNA
Figure 2. Adeno-ANT2 shRNA virus effectively induces apoptosis in cancer stem-like cells of a breast cancer cell line (stem-like cells). (A) CD44+/CD24- fractions were sorted from MDA-MB-231 and MCF7 cells using the MACS. The sorting purities were confirmed by FACS analysis. Cells were infected with adenoscramble shRNA virus or adenoANT2 shRNA virus. After $24 \mathrm{~h}$ of treatment with adeno-virus, cells were stained with AnnexinV and $\mathrm{PI}$ and analyzed by flow cytometry. (B) MCF10A cells were transfected with scramble shRNA or E-cadherin shRNA (each $1 \mu \mathrm{g}$ ). After $24 \mathrm{~h}$, cells were re-infected with adeno-scramble shRNA virus or adeno-ANT2 shRNA virus. After $24 \mathrm{~h}$ of treatment with adeno-virus, cells were stained with AnnexinV and PI and analyzed by flow cytometry.

\section{Results}

\section{ANT2 expression was high in breast cancer stem-like cells and non-stem-like cells of breast cancer cell lines, and knockdown of ANT2 by adeno-shRNA virus efficiently induced cell death}

To determine the levels of expression of ANT2 in both cancer stem-like cells and non- stem-like of a human breast cancer cell line, we first isolated progenitor cells based on the cell surface expression of CD44 and CD24 (Thompson et al., 1992; Gordon et al., 2003; Charafe-Jauffret et al., 2006; Elstrodt et al., 2006) and examined ANT2 mRNA levels in unsorted and sorted cell populations. The percentage of CD44+/CD24- cells was $>80 \%$ in MDA-MB-231 cells and $<10 \%$ in MCF7 cells (Supplementary Data 1). Moreover, aldehyde dehydrogenase (ALDH) activity was used as a functional marker for "stem-likeness" (data not shown). RT-PCR showed that ANT2 mRNA was highly expressed both in the mixed (unsorted) cell and sorted population (CD44+/CD24-), and a similar result was obtained by the real-time PCR method (Figure 1A). In addition, we sought to obtain a population of stem-like cells from a human mammary gland epithelial cell line (MCF10A) by inducing the population of stem-like cells to pass through an epithelial-mesenchymal transition (EMT). To this end, we modified MCF10A experimentally by shRNA-mediated inhibition of E-cadherin (MCF10A ${ }^{\mathrm{EMT}}$ ). Confirming previous reports, an E-cad shRNA triggered an EMT and resulted in acquisition of the mesenchymal phenotype (Figure 1B). Interestingly, ANT2 expression was induced and up-regulated in MCF10A cells with EMT induction (Figure 1B). Taken together, we demonstrated high expression of ANT2 in human breast cancer and mesenchymally transdifferentiated breast epithelial cells $\left(\right.$ MCF10A $^{\text {EMT }}$ ). Knockdown of ANT2 by adeno-shRNA virus (Supplementary Data 2) efficiently induced apoptotic cell death in CD44+/CD24-sorted stemlike cells of MDA-MB-231 and MCF7 (50-60\%), and MCF10A ${ }^{\text {EMT }}(30-40 \%)$ cells (Figures $2 \mathrm{~A}$ and $2 \mathrm{~B}$ ). These data indicated that ANT2 suppression by shRNA could be utilized for an effective therapy of breast cancer stem-like cells.

\section{Adeno-ANT2 shRNA virus suppressed tumor sphere formation of stem-like cells of breast cancer}

Tumor sphere (or mammosphere) formation is known as a characteristic feature of breast CSCs, and can be used for in vitro measurement of CSC activity. Adeno-ANT2 shRNA virus-treated progenitor cells had an approximate 10-fold decrease in tumor sphere-forming ability relative to adeno-scramble shRNA virus-treated stem-like cells (Figure 3 ). We also assayed the tumor sphere-forming ability of MCF10A ${ }^{\mathrm{EMT}}$ cells, but we did not obtain similar results. In our experiments, a single transfection of adeno-shRNAs achieved $>80 \%$ knockdown (Supplementary Data 2) that lasted 10-14 days post-transfection, then progressively diminished. 

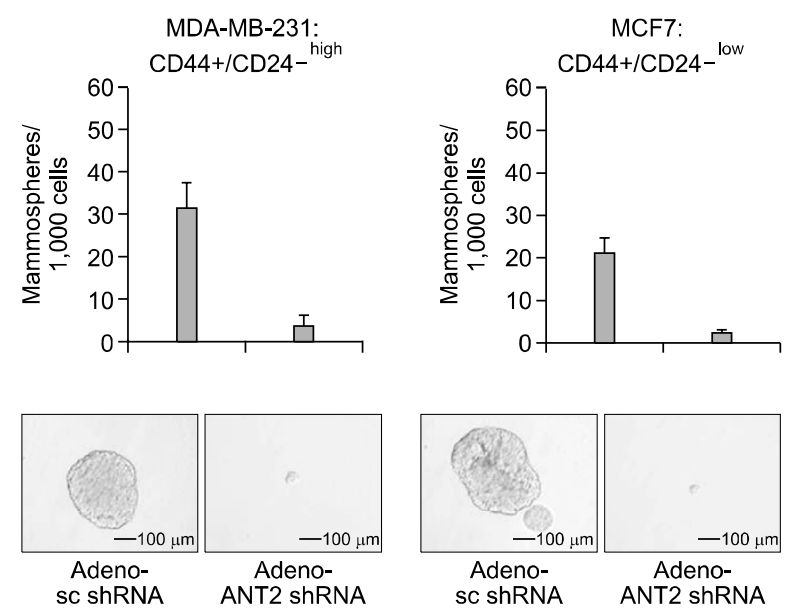

Figure 3. Adeno-ANT2 shRNA virus suppresses sphere formation of cancer stem-like cells of a breast cancer cell line. (A) CD44+/CD24- fractions were sorted from MDA-MB-231 and MCF7 cells using the MACS. The sorting purities were confirmed by FACS analysis. Cells were infected with adeno-scramble shRNA virus or adeno-ANT2 shRNA virus. After $24 \mathrm{~h}$, single-cell suspensions were plated $(30,000$ cells/well) in 6 -well ultra-low attachment plates in F12 $+5 \%$ FBS, insulin, and hydrocortisone. Mammospheres were cultured for 8 days, and those collected from non-adherent cultures were counted.

Taken together, these results implied that adenoANT2 shRNA virus suppressed the tumor sphereforming CSC activity of stem-like cells of breast cancer.

\section{Stem-like cells of breast cancer cell lines exhibited drug resistance, and adeno-ANT2 shRNA virus-enhanced chemosensitivity}

Treatment of cancer with chemotherapeutic agents has often led to an enrichment of the CSC population which has frequently shown drug resistance (Dean et al., 2005). We isolated stem-like cells, and examined their sensitivity to doxorubicin in unsorted and sorted cell populations. The percentage of CD44+/CD24- cells was higher in MDA-MB-231 $(80 \%)$ than MCF7 cells $(10 \%)$, and unsorted MDA-MB-231 cells were resistant to doxorubicin compared with MCF7 cells, which are doxorubicin-sensitive. However, in both cell lines, the stem-like cell population showed strong resistance to doxorubicin (Figures 4A and 4B). Accordingly, we assessed the chemosensitizing effects of adenoANT2 shRNA virus on unsorted and sorted (stem-like) cells, and showed that adeno- ANT2 shRNA virus markedly sensitized unsorted cells and sorted (stem-like cell) MDA-MB-231 and MCF7 cells to doxorubicin (Figures $4 \mathrm{~A}$ and $4 \mathrm{~B}$ ). We also showed that MCF10A ${ }^{\mathrm{E}-\mathrm{cad}}$ ShRNA cells were more resistant to doxorubicin than MCF10A ${ }^{\text {control shRNA }}$ cells and that adeno-ANT2 shRNA virus sensitized not only
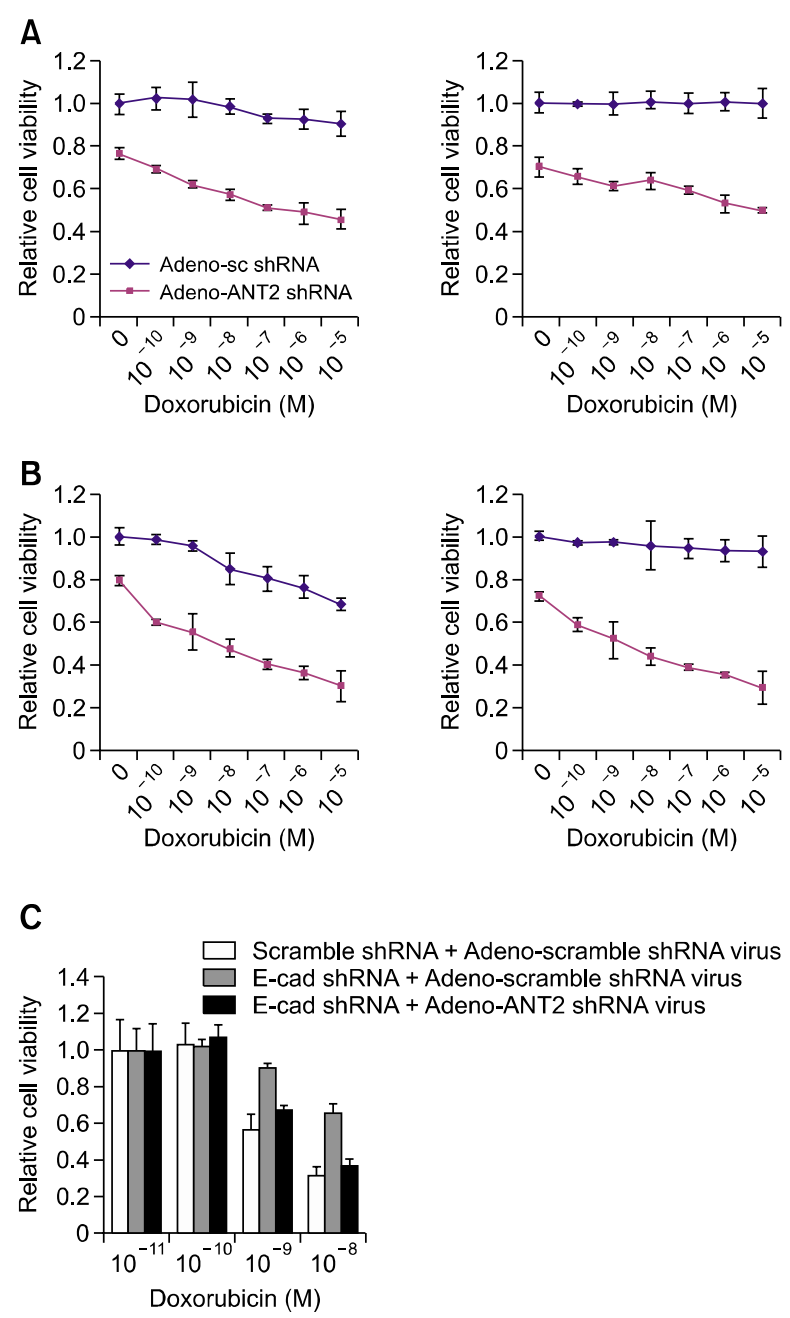

Figure 4. Adeno-ANT2 shRNA virus enhances chemosensitivity of progenitor cells of a breast cancer cell line. (A) CD44+/CD24- fractions were sorted from MDA-MB-231 using the MACS. The sorting purities were confirmed by FACS analysis. Unsorted or CD44+/CD24- sorted cells were infected with adeno-scramble shRNA virus or adeno-ANT2 shRNA virus. After $24 \mathrm{~h}$ of treatment with adeno-virus, cells were treated with doxorubicin, and $12 \mathrm{~h}$ later, a cytotoxicity assay of cells was performed using CCK8 assay kits. (B) CD44+/CD24- fractions were sorted from MCF7 using the MACS. The sorting purities were confirmed by FACS analysis. Unsorted or CD44+/CD24- sorted cells were infected with adeno-scramble shRNA virus or adeno-ANT2 shRNA virus. After $24 \mathrm{~h}$ of treatment with adeno-virus, cells were treated with doxorubicin, and $12 \mathrm{~h}$ later, a cytotoxicity assay of cells was performed using CCK8 assay kits. (C) MCF10A cells were transfected with scramble shRNA or E-cadherin shRNA (each $1 \mu \mathrm{g}$ ). After $24 \mathrm{~h}$, cells were re-infected with adeno-scramble shRNA virus or adeno-ANT2 shRNA virus. After $24 \mathrm{~h}$ of treatment with adeno-virus, cells were treated with doxorubicin, and $12 \mathrm{~h}$ later, a cytotoxicity assay of cells was performed using CCK8 assay kits.

MCF10A control shRNA $^{\text {, but also MCF10A }}$ E-cad shRNA $^{\text {to }}$ doxorubicin (Figure 4C). 
A

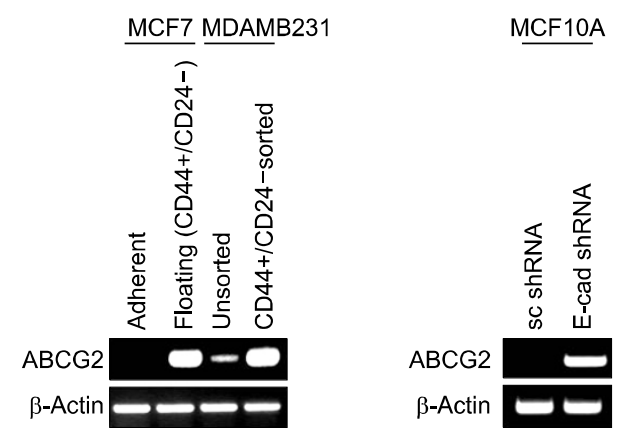

B

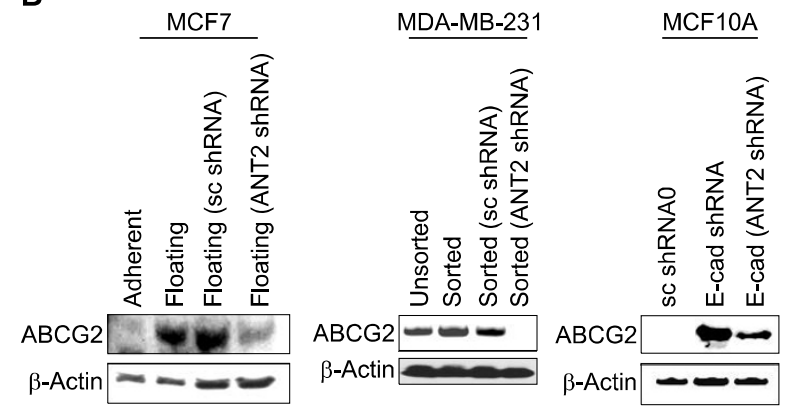

Figure 5. ABCG2 is highly expressed in cancer stem-like cells of a breast cancer cell line and down-regulated by ANT2 shRNA. (A) CD44+/CD24- fractions were sorted from MDA-MB-231 and MCF7 cells using the MACS. The sorting purities were confirmed by FACS analysis. RT-PCR to detect the levels of ABCG2 expression in unsorted and CD44+/CD24- sorted cells. Total RNA was extracted from respective cells and subjected to RT-PCR using specific primers for human ABCG2 or $\beta$-actin (internal control). MCF10A cells were transfected with scramble shRNA or E-cadherin shRNA (each $1 \mu \mathrm{g}$ ), and ABCG2 expression was detected by RT-PCR. (B) Western blotting for identifying the regulation of ABCG2 protein levels by adeno-ANT2 shRNA virus. Unsorted or CD44+/CD24- sorted cells were infected with adeno-scramble shRNA virus or adeno-ANT2 shRNA virus. After $24 \mathrm{~h}$ of treatment with adeno-virus, cell extracts were prepared for performing western blotting with anti-ABCG2 and $\beta$-actin antibodies. MCF10A cells were transfected with scramble shRNA or E-cadherin shRNA (each $1 \mu \mathrm{g}$ ), re-infected with adeno-ANT2 shRNA virus, and ABCG2 protein levels were detected by western blot.

\section{ABCG2 was highly expressed in stem-like cells of a breast cancer cell line, and the expression and activity of ABCG2 was down-regulated by adeno-ANT2 shRNA virus}

Several reports have suggested that CSCs have resistance to chemotherapy by expressing P-glycoprotein and ABCG2. CSCs therefore also have an ability to export Hoechst33342, which is a substrate for ABCG2. RT-PCR showed that ABCG2 was much more highly expressed in the sorted (CD44+/ CD24-) cancer stem-like cell population than the non-sorted mixed cell population (Figure $5 \mathrm{~A}$ ), which may be one of the causes leading to resistance to doxorubicin in cancer stem-like cells of MDA-MB-231 and MCF7 (Figure 4). Therefore, we tested if adenoANT2 shRNA virus had an effect on the regulation of ABCG2 expression. As shown in Figure 5B, ANT2 shRNA transfection reduced $A B C G 2$ expression of progenitor cells. Moreover, ABCB1 expression was also down-regulated (Supplementary Data 3). In the activity assay of ABCG2 measuring Hoechst33342 export (Figure 6), ANT2 shRNA treatment resulted in the intracellular accumulation of Hoechst33342 in cancer stem-like cells, indicating inhibition of ABCG2 activity by ANT2 shRNA. ABCG2 expression was not detected in MCF10A cells, but induced in MCF10A ${ }^{\text {EMT }}$ (or MCF10A ${ }^{\text {E-cad shRNA }}$ ) cells (Figure 5A). ANT2 shRNA also suppressed ABCG2 and its activity in MCF10A ${ }^{\mathrm{EMT}}$ cells (Figures $5 \mathrm{~B}$ and 6 ). Altogether, these results indicated that ANT2 shRNA might suppress the expression and activity of ABCG2 in breast cancer stem-like cells, thereby restoring sensitivity to doxorubicin.

\section{Discussion}

CSCs exhibit a generalized resistance to apoptosis, suggesting that they may not be possible in practice in the search for therapies that specifically target CSCs. Because CSCs are able to self-renew and regenerate tumors, similar to a primary tumor, they
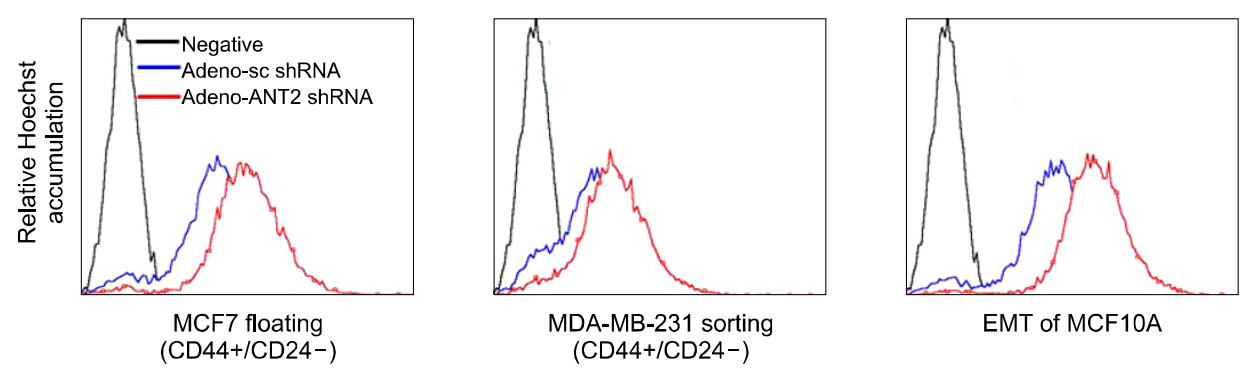

Figure 6. ANT2 shRNA inhibits ABCG2 activity in cancer stem-like cells of a breast cancer cell line. CD44+/CD24- population from MCF7 and MDA-MB-231 cells or E-cadherin shRNA-transfected MCF10A were infected with adeno-scramble shRNA virus or adeno-ANT2 shRNA virus. After $24 \mathrm{~h}$ of treatment with adeno-virus, cells were stained with Hoechst33342 and analyzed by flow cytometry for measurement of ABCG2 activity. 
have been thought to be associated with recurrence (Al-Hajj and Clarke, 2004; Ponti et al., 2005; Vermeulen et al., 2008). Therefore, it is important to study the characteristics of CSCs and to develop new therapies targeting them (Chiodi et al., 2011).

ANT2 has been viewed as an attractive cancer therapeutic target in breast cancer (Jang et al., 2008). We previously reported that plasmid vectorbased ANT2 RNA interference could be an efficient molecular therapeutic method for breast cancer which frequently shows high expression of ANT2. Moreover, knock-down of ANT2 by shRNA downregulated HER2/neu through suppression of HSP90 function and inhibited the PI3K/Akt signaling pathway, ultimately resulting in suppressed migration and invasion of breast cancer cells (Jang et al., 2010).

In the present study, we used SP (CD44 ${ }^{\text {high }}$ CD24-) cells derived from breast cancer cell lines as a representative cancer stem-like progenitor cells, observed ANT2 expression markedly up-regulated in both cancer stem-like cells and non-cancer stem-like cells of breast cancer cell lines, and that knock-down of ANT2 by RNA interference efficiently induced cell death of cancer stem-like cells as well as non- cancer stem-like cells. As another model of cancer stem-like cells, we induced epithelial and mesenchymal transition in MCF10A cells. MCF10A ${ }^{\text {EMT }}$ cells exhibited high expression of ANT2 unlike MCF10A cells, and shared some stem-cell like characteristics with SP cells in terms of chemoresistance and ABCG2 overexpression. These MCF10A ${ }^{\mathrm{EMT}}$ cells were also susceptible to ANT2 shRNA and cell death occurred.

In addition to inducing apoptotic cell death in cancer stem-like cells, adeno-ANT2 shRNA virus suppressed tumor sphere formation of cancer stem-like cells of breast cancer cell lines. Moreover, cancer stem-like cells of a breast cancer cell line exhibited increased drug (doxorubicin) resistance and concomitantly multi-drug resistant-related receptors, such as ABCG2, are highly expressed. ANT2 shRNA caused progenitor cells and MCF10A ${ }^{\text {EMT }}$ cells to overcome chemoresistance through the down-regulation of expression and activity of $A B C G 2$.

RNA interference is a phenomenon that can be used to block specific gene expression, and has had advantages over other strategies because of its high selectivity and potency. Moreover, transfection with synthetic dsRNA has been a highly useful method for the functional analysis of specific genes in vitro. However, dsRNA has been unsuitable for RNAi applications for genetic blockade in humans because of its low transfection rate and short duration of interference. DNA vector-based siRNA technology has had some advantages over ds-siRNA, as it induces more efficient and stable RNA interference. However, DNA plasmid vector-induced siRNA still has limitations with respect to the clinical application of siRNA technology due to its low transfection efficiency.

Adenoviruses have been widely used for cancer gene therapy because of their high gene transfer efficiency and levels of expression. Therefore, in the present study, we used an adenovirus expressing shRNA to ANT2. Adenoviruses have been used to induce RNA interference and here, we report for the first time that an adenovirus with an H1-RNA promoter effectively blocked p53 by expressing siRNA to p53. Further, transduction with several adenoviruses expressing siRNAs to certain target genes led to the efficient and specific reduction of specific mRNAs and encoding proteins (Shen et al., 2003). Moreover, conditionally replicating adenovirus (CRAD) expressed short hairpin RNA was also shown to reduce target gene expression

In conclusion, we believe that adenoviruses expressing shRNA to ANT2 can be utilized in anticancer therapy of breast cancer-stem like cells, and will have future use as a chemotherapy-sensitizing agent targeting breast cancer-stem like cells. We will extend the current investigation of adenovirus-shRNA to ANT2 using different cancer models.

\section{Methods}

\section{Cell culture}

The human breast carcinoma cell lines, MCF7 and MDA-MB-231, were used throughout this study. These cells were provided by the Korean Cell Line Bank (Seoul, Korea) and were cultured in DMEM supplemented with $10 \%$ FBS, $100 \mathrm{u} / \mathrm{ml}$ of penicillin, and $100 \mu \mathrm{g} / \mathrm{ml}$ of streptomycin. MCF-10A, a line of healthy epithelial cells from human mammary gland (CRL-10317), which provided by the American Type Culture Collection (Manassas, VA) was used as a control. This cell line was maintained in DMEM/Ham Nutrient Mixture F-12 (1:1) with the addition of epidermal growth factor $(20 \mathrm{ng} / \mathrm{ml})$, cholera enterotoxin $(100 \mathrm{ng} / \mathrm{ml})$, insulin $(10 \mu \mathrm{g} / \mathrm{ml})$, and hydrocortisone (500 $\mathrm{ng} / \mathrm{ml}$ ) in the presence of $5 \%$ horse serum.

\section{Sorting of cancer stem-like cells (sub-population; SP)}

To allow for the isolation of CD44+/CD24- cell populations, human breast cancer cell lines (MCF7 and MDA-MB-231) were sorted by magnetic cell sorting (MACS; Miltenyi Biotech, Auburn, CA) for CD44+ cells prior to SP sorting. In MACS, cells were resuspended in $0.1 \%$ bovine serum albumin (BSA)-PBS and labeled with phycoerythrin (PE)conjugated CD44 antibody (BD-PharMingen, San Diego, California). After addition of the primary antibody, the cells 
were incubated with anti-PE microbeads (Miltenyi Biotec, Bergisch Gladbach, Germany). The cell suspension was filtered then sorted using the "DOUBLE POSITIVE SORT" program of an AutoMACS system (Miltenyi Biotech). Both the positive and negative fractions were analyzed by FACS to assess sorting efficiency using a FACSCalibur flow cytometer (Becton Dickenson, Mountain View, CA). The selected human breast cancer cell lines were then sorted for SP.

\section{Generation of adenoviruses expressing shRNA to ANT2}

ANT2 shRNA (dsDNA with sense-loop-antisense) were cloned into pSilencer H1. The target of ANT2 shRNA was complementary to exon 2 accession number NM001152, and the sequence was 5'-GCAGAUCACUGCAGAUAAGTT-3'. The adenoviruses were constructed using the BD AdenoXTM expression system (BD Biosciences Clontech, Palo Alto, CA). Cloned pRNAT was digested with I-Ceul and $\mathrm{PI}-\mathrm{Scel}$, and ligated with pre-digested $\mathrm{BD}$ adeno-X viral DNA (32.6 kb). Following Swal digestion, adenoviral DNA was prepared on a large scale in Escherichia coli, and the adenoviral DNA was then transfected into low-passage HEK293 cells after Pacl digestion. Adenovirus generation was confirmed by the appearance of a cytopathic effect. The adenoviruses were propagated in HEK293 cells and purified using a BD Adeno-XTM purification kit (BD Biosciences Clontech). Viral titers were determined using a BD Adeno XTM rapid titer kit (BD Biosciences Clontech).

\section{Transfection}

For transfection, cells were plated on 6 -well plates $\left(2 \times 10^{5}\right.$ cells per well) or $100-\mathrm{mm}$ dishes $\left(2 \times 10^{6}\right.$ cells), and were allowed to adhere for $24 \mathrm{~h}$. Lipofectamine 2000 (Invitrogen, Carlsbad, CA) was used for the transfections. pSilencer ${ }^{\mathrm{TM}}$ $3.1-\mathrm{H} 1$ puro ANT2 siRNA vectors or pSilencer $^{T \mathrm{M}} 3.1-\mathrm{H} 1$ puro scramble siRNA vectors were transfected into the cells. Transfected cells were then cultured for $4 \mathrm{~h}$ and the culture media were then replaced with fresh media supplemented with $10 \%$ FBS. The cells were harvested $24-48 \mathrm{~h}$ after transfection. MCF10A cells expressing either control shRNA (control shRNA) or shRNA targeting E-cadherin (E-cad shRNA) were generated with Lipofectamine 2000 (vide supra). Knock-down effect of shRNA was confirmed in all experiments (data not shown).

\section{RT-PCR}

Total RNA was extracted using Trizol (Invitrogen) according to the manufacturer's instructions. For RT-PCR analysis, $5 \mu \mathrm{g}$ of total RNA was reverse-transcribed using RT-PCR kits (Promega, Madison, WI). PCR was used to amplify target cDNA under the following conditions: 35 cycles of $94^{\circ} \mathrm{C}$ for $1 \mathrm{~min} ; 55^{\circ} \mathrm{C}$ for $1 \mathrm{~min}$; and $72^{\circ} \mathrm{C}$ for $2 \mathrm{~min}$. The PCR products were analyzed using standard agarose gel electrophoresis. The primers used for RT-PCR were as follows: ANT2 forward, 5'-CCGCAGCGCCGGAGTCAAA-3' and reverse, 5'-AGTCTGTCAAGAATGCTCAA-3'; E-cadherin forward, 5'-GACGCGGACGATGAT GTGAAC-3' and reverse, 5'-TTGTACGTGGTGGGATTGAAGA-3'; ABCG2 forward, 5'-CCATAGCCACAGGCCAAAGT-3' and reverse, 5'-GGGCCACATGATTCTTCCAC-3'; and $\beta$-actin forward, 5'-GGAAATCGTGCGTGACATTAAGG-3' and reverse, 5'-GGCTTTTAGGATGGCAAG GGA C-3'.

\section{Real-time PCR}

Real-time PCR was performed using an IQ5 thermal cycler (Bio-Rad, Hercules, CA) in the presence of SYBR-green. The optimization of the real-time PCR reaction was performed according to the manufacturer's instructions (User Bulletin 2 applied to the SYBR-Green I core reagent protocol), but scaled down to $25 \mu$ per reaction. The PCR conditions were standard (SYBR-Green I core reagent proto$\mathrm{col})$ and all reagents were provided in the SYBR-Green I core reagent kit, including AmpliTaq-polymerase (PE Applied-Biosystems). After optimization (see Results section), nucleotide primers were used at various concentrations for the detection and quantification of signal $\beta$-actin and coding ANT2.

\section{Mammosphere formation}

To generate mammospheres, single-cell suspensions were plated in $100 \Phi$ dish ultra-low attachment plates $(30,000$ cells/well; Corning, NY) in serum-free MEM supplemented with $20 \mathrm{ng} / \mathrm{ml} \mathrm{bFGF,} 20 \mathrm{ng} / \mathrm{ml} \mathrm{EGF}$, and B27 (all from Invitrogen). Mammospheres were cultured for 8 days, and those collected from non-adherent cultures were quantified with a Multisizer 3 Coulter Counter (sizing range, 14-336 $\mu \mathrm{m})$.

\section{Cell viability assay}

The viabilities of treated cells were measured using a Cell Counting Kit (CCK-8; Dojindo Molecular Technologies, Kumamoto, Japan). All assays were performed in triplicate, and the results were presented as the mean values of three replicate experiments.

\section{Western-blot}

For western blot analyses, cells were harvested and were lysed with lysis buffer $(5 \mathrm{mM} / \mathrm{L}$ ethylenediamine tetraacetic acid, $300 \mathrm{mM} / \mathrm{L} \mathrm{NaCl}, 0.1 \% \mathrm{NP}-40,0.5 \mathrm{mM} / \mathrm{L} \mathrm{NaF}, 0.5$ $\mathrm{mM} / \mathrm{L} \mathrm{Na} \mathrm{VO}_{4}, 0.5 \mathrm{mM} / \mathrm{L}$ phenylmethylsulfonyl fluoride, and $10 \mu \mathrm{g} / \mathrm{ml}$ each of aprotinin, pepstatin, and leupeptin; Sigma, St Louis, MO). After centrifugation at 15,000 $\times g$ for $30 \mathrm{~min}$, the concentrations of supernatant proteins were analyzed by a Bradford reagent (Bio-Rad). For analysis of protein content, $50 \mu \mathrm{g}$ of total proteins was electrophoresed in $10 \%$ SDS-PAGE gel, transferred to polyvinylidene difluoride membranes (Millipore, Bedford, MA), and were then incubated with the respective antibodies, as indicated above. Immunoblots were visualized using an enhanced chemiluminescence detection system (Amersham Pharmacia Biotech, Uppsala, Sweden). Anti-ANT2, antiABCG2, and anti- $\alpha$-tubulin antibodies were obtained from Santa Cruz Biotechnology, Inc. (Santa Cruz, CA). 


\section{Apoptosis assay}

Apoptotic cell death was assessed by flow cytometry assay using propidium iodide (PI) staining and Annexin-V fluorescein isothiocyanate (FITC) and $\mathrm{PI}$ double-staining. The flow cytometry assay using PI staining was performed as described in the section on cell cycle analysis. Annexin-V and PI double-staining was performed using an Annexin-V FITC Apoptosis Detection Kit (R\&D Systems, Abingdon, UK), following the manufacturer's protocol. Each group was stained and analyzed by a flow cytometer.

\section{FACS (surface staining)}

Surface staining was performed with anti-ABCG2 and FITC-conjugated anti-mouse IgG antibodies, then analyzed by flow cytometry (Epics XL; Coulter, CA).

\section{FACS (Hoechst 33342 staining)}

Hoechst 33342 (Molecular Probes, Eugene, OR) was added to a concentration of $2-5 \mu \mathrm{g} / \mathrm{ml}$. Cells were incubated at $37^{\circ} \mathrm{C}$ for $90 \mathrm{~min}$, then placed on ice until analysis. Fluorescence from the Hoechst-stained cells was excited by $100-150 \mathrm{~mW}$ UV from an Innova 305c argon ion laser (Coherent, Santa Clara, CA). Fluorescence was monitored at 2 emission bands from 400-480 nm (blue) and 690-800 $\mathrm{nm}$ (red).

\section{Statistical analysis}

Data were analyzed using the Student's $t$ test, and differences are considered statistically significant at $P<0.05$.

\section{Supplemental data}

Supplemental data include three figures, and can be found with this article online at http://e-emm.or.kr/article/article files/SP-44-4-01.pdf.

\section{Acknowledgements}

This work was supported in part by grants from Ministry of Knowledge Economy (\#10035577).

\section{Competing interests}

The authors have applied for a domestic patent and will apply for an international patent regarding the utilization of ANT2 siRNA technology as a therapeutic method for cancer treatment. Seoul National University College of Medicine will retain the patent. The authors declare that they have no other competing interests.

\section{Authors' contributions}

$\mathrm{J}-\mathrm{YJ}$ performed the majority of the experiments and was responsible for generating the results, the data analysis, and preparing the manuscript. M-KK contributed to the construction of adenovirus shRNA to ANT2. Y-KJ was also responsible for analysis of the data, as well as writing the manuscript. C-WK contributed to the design of the project data analysis, and writing the manuscript. All authors reviewed and agreed to the content of the final, submitted version of the manuscript.

\section{References}

Al-Hajj M, Clarke MF. Self-renewal and solid tumor stem cells. Oncogene 2004;23:7274-82

Charafe-Jauffret E, Ginestier C, Monville F, Finetti P, Adelaide J, Cervera N, Fekairi S, Xerri L, Jacquemier J, Birnbaum D, Bertucci F. Gene expression profiling of breast cell lines identifies potential new basal markers. Oncogene 2006;25:2273-84

Chevrollier A, Loiseau D, Chabi B, Renier G, Donay O, Malthiery Y, Stepien G. ANT2 isoform required for cancer cell glycolysis. J Bioenerg Biomembr 2005a;37:307-16

Chevrollier A, Loiseau D, Gautier F, Malthlery Y, Stepien G. ANT2 expression under hypoxic conditions produces opposite cellcycle behavior in 143B and HepG2 cancer cells. Mol Carcinog 2005b;42:1-8

Chevrollier A, Loiseau D, Stepien G. What is the specific role of ANT2 in cancer cells? Med Sci (Paris) 2005c;21:156-61

Chiodi I, Belgiovine C, Donà F, Scovassi I, Mondello C. Drug treatment of cancer cell lines: a way to select for cancer stem cells? Cancers 2011;3:1111-28

Dean M, Fojo T, Bates S. Tumour stem cells and drug resistance. Nat Rev 2005;5:275-84

Doerner A, Pauschinger M, Badorff A, Noutsias M, Giessn $\mathrm{S}$, Schulze K, Bilger J, Rauch U, Schultheiss HP. Tissuespecific transcription pattern of the adenine nucleotide translocase isoforms in humans. FEBS Lett 1997;414: 258-62

Elstrodt F, Hollestelle A, Nagel JH, Gorin M, Wasielewski M, van den Ouweland A, Merajver SD, Ethier SP, Schutte M. BRCA1 mutation analysis of 41 human breast cancer cell lines reveals three new deleterious mutants. Cancer Res 2006;66:41-5

Faure Vigny H, Heddi A, Giraud S, Chautard D, Stepien G. Expression of oxidative phosphorylation genens in renal tumors and tumoral cell lines. Mol Carcinog 1996;16:165-72

Gordon LA, Mulligan KT, Maxwell-Jones H, Adams M, Walker RA, Jones JL. Breast cell invasive potential relates to the myoepithelial phenotype. Int J Cancer 2003;106:8-16

Gross A, McDonnell JM, Korsmeyer SJ. BCL-2 family members and the mitochondria in apoptosis. Genes Dev 1999;13:1899-911

Hermann PC, Huber SL, Herrler T, Aicher A, Ellwart JW, Guba M, Bruns CJ, Heeschen C. Distinct populations of cancer stem cells determine tumor growth and metastatic activity in human pancreatic cancer. Cell Stem Cell 2007;1: 313-23

Jang JY, Choi Y, Jeon YK, Kim CW. Suppression of adenine nucleotide translocase-2 by vector based siRNA in human breast cancer cells induces apoptosis and inhibits tumor 
growth in vitro and in vivo. Breast Cancer Res 2008;10:R11

Jang JY, Jeon YK, Kim CW. Degradation of HER2/neu by ANT2 shRNA suppresses migration and invasiveness of breast cancer cells. BMC Cancer 2010;10:391

Kong D, Li Y, Wang Z, Sarkar FH. Cancer stem cells and epithelialto-mesenchymal transition (EMT)-phenotypic cells: are they cousins or twins? Cancers 2011;3:716-29

Le Bras M, Borgne-Sanchez A, Touat Z, EI Dein OS, Deniaud $A$, Maillier E, Lecellier G, Rebouillat D, Lemair C, Kroemer $\mathrm{G}$, Jacotot $\mathrm{E}$, Brenner $\mathrm{C}$. Chemosensitization by knockdown of adenine nucleotide translocase-2. Cancer Res 2006;66: 9143-52

Liu G, Yuan X, Zeng Z, Tunici P, Ng H, Abdulkadir IR, Lu L, Irvin D, Black KL, Yu JS. Analysis of gene expression and chemoresistance of $\mathrm{CD}_{133^{+}}$cancer stem cells in glioblastoma. Mol Cancer 2006;5:67

Lou H, Dean M. Targeted therapy for cancer stem cells: the patched pathway and $A B C$ transporters. Oncogene 2007; 26;1357-60

Luciakova K, Barath P, Poliakova D, Persson A, Nelson BD. Repression of the human adenine nucleotide translocase-2 gene in growth-arrested human diploid cells. J Biol Chem 2003;278:30624-33

Ma S, Lee TK, Zheng BJ, Chan KW, Guan XY. CD133+ HCC cancer stem cells confer chemoresistance by preferential expression of the Akt/PKB survival pathway. Oncogene 2008;27:1749-58

Morel AP, Lièvre M, Thomas C, Hinkal G, Ansieau S, Puisieux A. Generation of breast cancer stem cells through epithelialmesenchymal transition. PLoS One 2008;3:e2888

Pearce DJ, Bonnet D. The combined use of Hoechst efflux ability and aldehyde dehydrogenase activity to identify murine and human hematopoietic stem cells. Exp Hematol 2007;35:1437-46

Phillips TM, McBride WH, Pajonk F. The response of $\mathrm{CD} 24^{(- \text {llow })} / \mathrm{CD} 44^{+}$breast cancer-initiating cells to radiation. $J$ Natl Cancer Inst 2006;98:1777-85

Ponti D, Costa A, Zaffaroni N, Pratesi G, Petrangolini G, Coradini D, Pilotti S, Pierotti MA, Daidone MG. Isolation and in vitro propagation of tumorigenic breast cancer cells with stem/progenitor cell properties. Cancer Res 2005;65: 5506-11

Schinkel AH, Jonker JW. Mammalian drug efflux transporters of the ATP binding cassette $(A B C)$ family: an overview. Adv Drug Deliv Rev 2003;55:3-29
Shen C, Buck AK, Liu X, Winkler M, Reske SN. Gene silencing by adenovirus delivered siRNA. FEBS Letters 2003;539:111-4

Sheridan C, Kishimoto H, Fuchs RK, Mehrotra S, Bhat-Nakshatri P, Turner $\mathrm{CH}$, Goulet R Jr, Badve S, Nakshatri $\mathrm{H}$. CD44 $4^{+} \mathrm{CD} 24^{-}$breast cancer cells exhibit enhanced invasive properties: an early step necessary for metastasis. Breast Cancer Res 2006;8:R59

Shipitsin M, Campbell LL, Argani P, Weremowicz S, Bloushtain-Qimron N, Yao J, Nikolskaya T, Serebryiskaya T, Beroukhim R, Hu M, Halushka MK, Sukumar S, Parker LM, Anderson KS, Harris LN, Garber JE, Richardson AL, Schnitt SJ, Nikolsky Y, Gelman RS, Polyak K. Molecular definition of breast tumor heterogeneity. Cancer Cell 2007;11:259-73

Stepien G, Torroni A, Chung AB, Hodge JA, Wallace DC. Differential expression of adenine nucleotide translocator isoforms in mammalian tissues and during muscle cell differentiation. J Biol Chem 1992;267:14592-7

Suliman A, Lam A, Datta R, Srivastava RK. Intracellular mechanisms of TRAIL: apoptosis through mitochondrialdependent and -independent pathways. Oncogene 2001; 20:2122-33

Thompson EW, Paik S, Brunner N, Sommers CL, Zugmaier G, Clarke R, Shima TB, Torri J, Donahue S, Lippman ME. Association of increased basement membrane invasiveness with absence of estrogen receptor and expression of vimentin in human breast cancer cell lines. J Cell Physiol 1992;150:534-44

Todaro M, Alea MP, Di Stefano AB, Cammareri P, Vermeulen L, lovino F, Tripodo C, Russo A, Gulotta G, Medema JP, Stassi $\mathrm{G}$. Colon cancer stem cells dictate tumor growth and resist cell death by production of interleukin-4. Cell Stem Cell 2007;1:389-402

Vermeulen L, Sprick MR, Kemper K, Stassi G, Medema JP. Cancer stem cells-old concepts, new insights. Cell Death Differ 2008;15:947-58

Wulf GG, Wang RY, Kuehnle I, Weidner D, Marini F, Brenner MK, Andreeff M, Goodell MA. A leukemic stem cell with intrinsic drug efflux capacity in acute myeloid leukemia. Blood 2001;98:1166-73

Zhou S, Schuetz JD, Bunting KD, Colapietro AM, Sampath J, Morris JJ, Lagutina I, Grosveld GC, Osawa M, Nakauchi $H$, Sorrentino BP. The ABC transporter Bcrp1/ABCG2 is expressed in a wide variety of stem cells and is a molecular determinant of the side-population phenotype. Nat Med 2001;7:1028-34 